\title{
Single-Mode Fiber and Few-Mode Fiber Photonic Lanterns performance evaluated for use in a Scalable Real-time Photon Counting Ground Receiver
}

\author{
Sarah A. Tedder*a , Brian E. Vyhnalek ${ }^{\mathrm{a}}$, Sergio Leon-Saval ${ }^{\mathrm{b}}$, Christopher Betters $^{\mathrm{b}}$, Bertram Floyd $^{\mathrm{c}}$,
} Jeremy Staffa ${ }^{\mathrm{d}}$, and Robert Lafon ${ }^{\mathrm{e}}$

${ }^{a}$ NASA Glenn Research Center, 21000 Brookpark Rd, Cleveland, OH, USA 44135-3127;

bInstitute of Photonics and Optical Science, University of Sydney, NSW 2006, Sydney, Australia;

${ }^{c}$ Hx5 Sierra, 21000 Brookpark Rd, Cleveland, OH, USA 44135-3127;

${ }^{\mathrm{d} U n i v e r s i t y ~ o f ~ R o c h e s t e r, ~ R o c h e s t e r, ~ N Y, ~ U S A ; ~}$

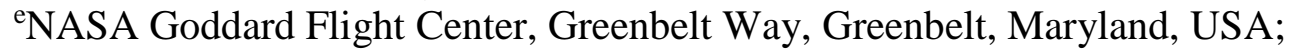

\begin{abstract}
Photonic lanterns provide an efficient way of coupling light from a single large-core fiber to multiple small-core fibers. This capability is of interest for space to ground communication applications. In these applications, the optical ground receivers require high-efficiency coupling from an atmospherically distorted focus spot to multiple fiber coupled single pixel super-conducting nanowire detectors. This paper will explore the use of photonic lanterns in a real-time ground receiver that is scalable and constructed with commercial parts.

The number of small-core fibers (i.e. an array of single or few-mode cores) that make a photonic lantern determines the number of spatial modes that they couple at the larger multimode fiber core end. For instance, lanterns made with $n$ number of single-mode fibers can couple $n$ number of spatial modes. Although the laser transmitted from a spacecraft originates as a Gaussian shape, the atmosphere distorts the beam profile by scrambling the phase and scattering energy into higher-order spatial modes. Therefore, if a ground receiver is sized for a target data rate with $n$ number of detectors, the corresponding lantern made with single-mode fibers will couple $n$ number of spatial modes. Most of the energy of the transmitted beam scattered into spatial modes higher than $n$ will be lost. This paper shows this loss may be reduced by making lanterns with few-mode fibers instead of single-mode fibers, increasing the number of spatial modes that can be coupled and therefore increasing the coupling efficiency to single pixel, single photon detectors. The free space to fiber coupling efficiency of these two types of photonic lanterns are compared over a range of the free-space coupling numerical apertures and mode field diameters. Results indicate the few mode fiber lantern has higher coupling efficiency for telescopes with longer focal lengths under higher turbulent conditions. Also presented is analysis of the jitter added to the system by the lanterns, showing the few-mode fiber photonic lantern adds more jitter than the single-mode fiber lantern, but less than a multimode fiber.
\end{abstract}

Keywords: Photonic lanterns, fiber coupling, free-space optical communications, photon counting, and ground receiver.

\section{INTRODUCTION}

Real-time photon counting ground receivers are needed to enable optical space to earth communications for both public and private applications. NASA is working on a scalable design assembled from commercial-off-the-shelf (COTS) components for the aft part of a ground receiver that includes the hardware and software that exists behind a collection telescope. The hardware includes the aft collection optics that delivers the light from the telescope to the detectors, superconducting nanowire detectors, cryostat, electronics, real-time field programmable gate array (FPGA) based receiver, and optical waveform decoder that is compliant to the standard being formed by the Consultative Committee for Space Data Systems [1]. The goal is to create one conceptual design that can be used for a variety of: data rates (up to $528 \mathrm{MB} / \mathrm{s}$ ), telescope aperture sizes, and environmental factors (background light and atmospheric turbulence levels). A scalable ground receiver has the potential to lower the cost by using similar equipment and software for multiple applications. Scalable receivers could also enable ground stations to scale up their data rate capability by expanding on existing

*sarah.a.tedder@nasa.gov; 
architecture. To implement this scalable ground receiver, NASA is considering a wide range of components already in use for other types of applications. This paper explores the use of a photonic lantern [2], originating from the field of astronomy, in a scalable receiver concept.

The scalable receiver concept under consideration in this paper and Refs. [3 and 4] uses a COTS array of individually coupled single-pixel superconducting nanowire single-photon detectors (SNSPDs). Using multiple detectors reduces blocking loss caused by detector reset time (or dead time) and increases the data rate achievable by the system. These SNSPDs are 14 micron in diameter and are butt-coupled with a single-mode or few-mode fibers. The efficiency of this coupling is discussed in detail in Ref. [4].

This paper focuses on a possible solution to collect the light from the telescope and deliver it to the multiple single-mode fiber (SMF) or few-mode fiber (FMF) coupled COTS SNSPDs. Although the laser transmitted from a spacecraft originates as a Gaussian shape, the light exiting from the telescope is multi-moded due to the atmospheric turbulence that distorts the beam profile by scattering energy and phase into higher-order spatial modes. Therefore a solution is needed to efficiently collect multi-mode light and split it to multiple SMF or FMFs. Photonic lanterns have this capability having been created to solve a similar problem in the astronomical field - to increase the coupling efficiency of light collected aft of telescopes and deliver it to multiple single-mode fiber Bragg gratings ${ }^{2}$. Their use for optical communication receivers has been explored in references [5-8].

Photonic lanterns are traditionally made from multiple SMFs' ${ }^{2}$. The number of modes collected by a lantern is scaled by the number of the SMFs that make up the lantern. To detect all the light collected by the lantern, each SMF must be coupled to an individual detector. Therefore, as atmospheric turbulence increases and the energy is scattered into higher order spatial modes, the number of detectors needed increases accordingly. Depending on the condition and desired data rate, the number of detectors needed could be higher than the number of detectors required to compensate for dead time. This would cause the cost of the receiver to increase. Therefore to reduce cost, a solution is needed to increase the mode coupling capacity of a lantern without increasing the number of output legs. A new approach to fabricate highly-multimoded lanterns with multi-core fibers was recently demonstrated [9], showing for the first time that it is possible to have multi-mode to few-mode (i.e. few single-mode multicore arrays) lantern transitions with high efficiency. Thus, a new type of lantern is considered for this application, one fabricated with few-mode fibers (FMFs) instead of SMFs.

This paper evaluates and compares the performance of a 7:1 FMF lantern and a 7:1 SMF lantern. The 7:1 FMF lantern is fabricated with FMFs that each allow 6 fiber spatial modes. These FMFs were selected because they still have smaller and similar mode field diameters to SMFs, allowing efficient coupling to the COTS SNSPDs as shown in Ref. [4]. In theory, this FMF lantern should be able couple a total of 42 modes, 6 times more than a 7:1 SMF lantern. Hence, the 7:1 FMF lantern has the potential to increase the mode coupling capacity, while still requiring same number of detectors as a 7:1 SMF lantern.

This paper presents measurements of the mode coupling efficiency of a 7:1 SMF photonic lantern and a 7:1 FMF photonic lantern across the first 21 fiber spatial modes. To enable evaluation of the telescope, the free space to fiber coupling efficiency of the lanterns measured over a range of numerical apertures (NAs) and mode field diameters is presented. Unfortunately, while increasing the number of allowed modes in the lantern increases the efficiency, it also has the potential to increase system jitter, thus limiting the receivable data rate of the system. Jitter of the two lanterns types is analyzed and compared in this paper. Finally, to minimize the blocking loss, the light must be distributed to each detector evenly. This paper presents measurements of how evenly the two types of lanterns split the light between their fiber output legs for a few example input spatial fiber mode profiles.

\section{EXPERIMENTAL SETUP}

The efficiency data presented in this paper was collected using the optical setup shown in Figure 1. As shown in the figure, a linear polarized fiber coupled $1550 \mathrm{~nm}$ laser was used as the light source. The light exiting the fiber is collimated with a $15 \mathrm{~mm}$ focal length reflective collimator. Depending on the targeted NA, a $2 \times$ beam expander was used (or not used) to increase the size of the laser beam. The linear polarization direction of the light was then aligned to the spatial light modulator's (SLM) polarization sensitivity axis. The light is then passed through a 50/50 beam splitter with half of the light reaching SLM's $12.8 \times 12.8 \mathrm{~mm}$ surface comprised of an array of $512 \times 512$ active liquid crystal pixels. Next, the SLM reflects the light back to the 50/50 beam splitter sending half of that energy into the focusing lens. To test a range of 
NAs, lenses with a range of focal lengths were used as follows: $8 \mathrm{~mm}, 11 \mathrm{~mm}, 18.4 \mathrm{~mm}$, and $25 \mathrm{~mm}$. The test fiber or lantern is placed at the focal point of the focusing lens with a three dimensional fine positioner. To determine the efficiency, the power is measured with a power meter having a $9.7 \times 9.7 \mathrm{~mm}$ active surface in two locations - just after the focusing lens and at the exit of the fiber or lantern.

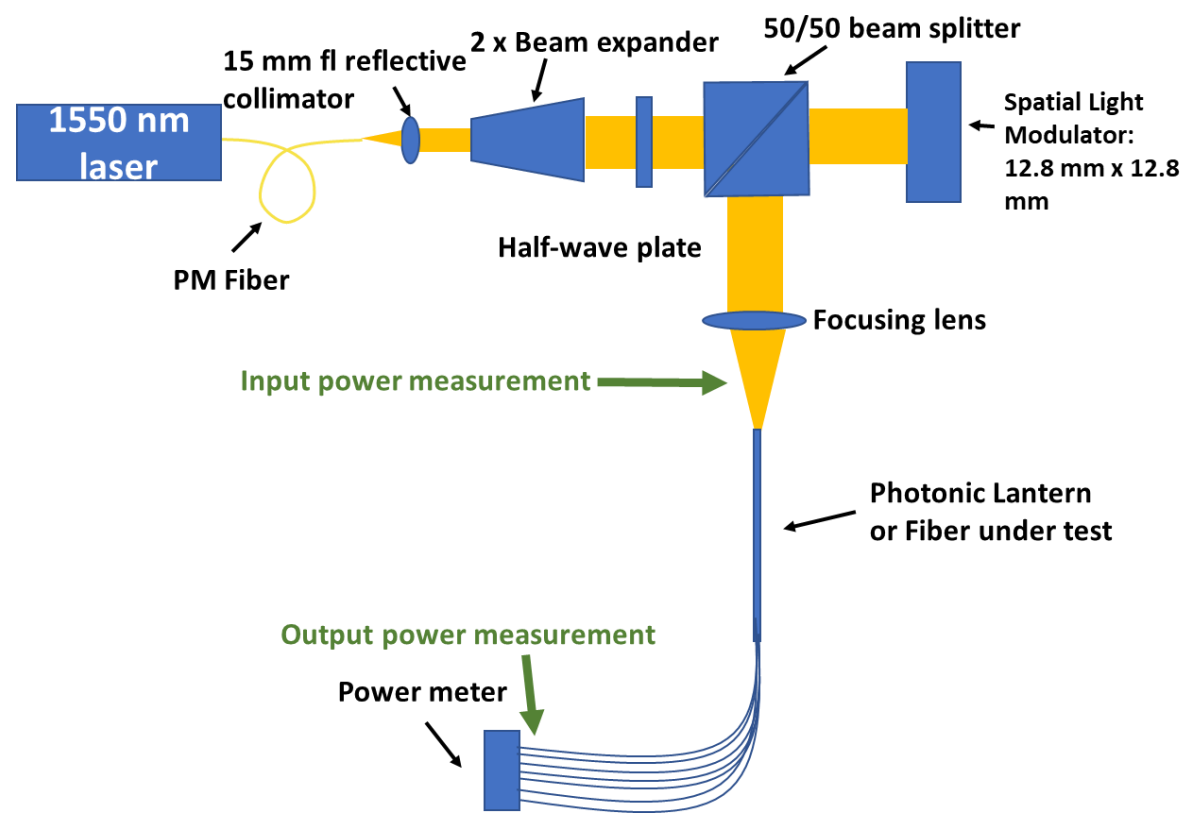

Figure 1: Schematic of the experimental setup for measuring the coupling efficiency of each device (not to scale)

\subsection{Test Photonic Lanterns and Fibers}

Two types of photonic lanterns were fabricated for this paper, one made with SMFs and one made with FMFs. For this application, the light is coupled into the MMF side of the lantern and split to 7 SMFs that are butt coupled on the detectors. A drawing of the cross section of the SMF photonic lantern is shown in Figure 2. The physical dimensions of this lantern are the summarized in Table 1.

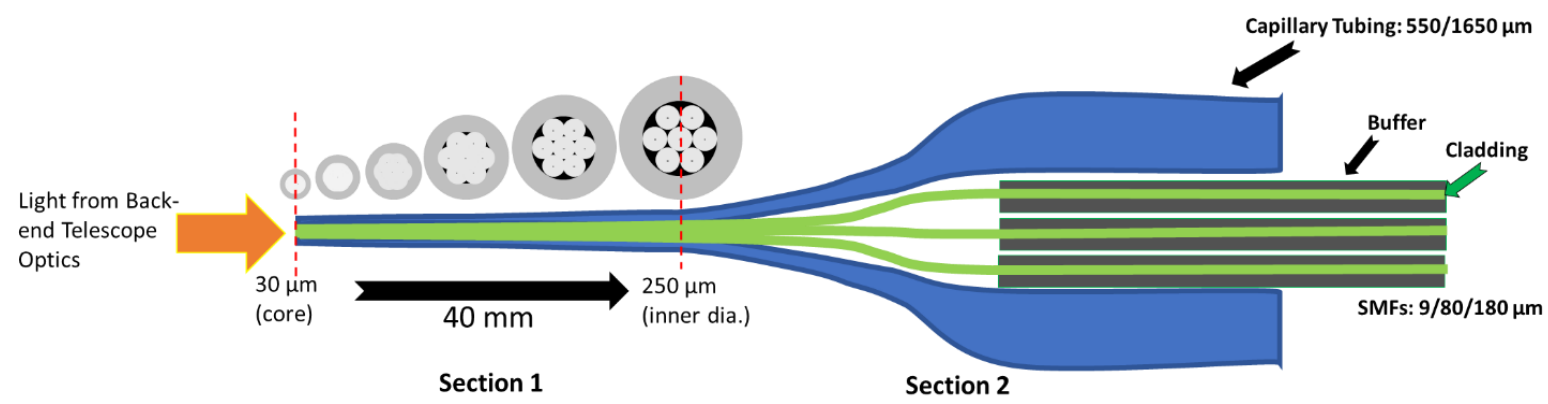

Figure 2: Cross-section illustration of the SMF photonic lantern (not to scale).

Table 1 also includes the specifications of the other fibers measured or analyzed for performance comparison. The lanterns were designed to match as many parameters to each other as practical. The SMF lantern was designed to have a 30 micron core with an NA of 0.09. The lantern transitions from a MMF input to $7 \mathrm{SMFs}$ in a $40 \mathrm{~mm}$ long taper, labeled as section 1 in Fig. 2. The remainder of the length of the lantern in the system is 7 SMF output legs, labeled section 2 in Fig. 2. The 7:1 SMF lantern was made in-house at Glenn Research Center with a fiber processing machine using 7 SMF that had NAs 
of 0.11-0.13. The SMF lantern can couple the first 7 fiber spatial modes: $\mathrm{LP}_{01}, \mathrm{LP}_{11 \mathrm{a}}, \mathrm{LP}_{11 \mathrm{~b}}, \mathrm{LP}_{21 \mathrm{a}}, \mathrm{LP}_{21 \mathrm{~b}}, \mathrm{LP}_{02}$, and $\mathrm{LP}_{31 \mathrm{a}}$ as shown in Ref. [10]. The FMF lantern is made with 7 graded index FMFs that each allow 6 spatial modes, $\mathrm{LP}_{01}, \mathrm{LP}_{11 a}$, $\mathrm{LP}_{11 \mathrm{~b}}, \mathrm{LP}_{21 \mathrm{a}}, \mathrm{LP}_{21 \mathrm{~b}}$, and $\mathrm{LP}_{02}$. The FMF lantern has a design NA of 0.15 . The number of modes the FMF lantern can couple is not yet fully understood, but exploration of this is presented in the results and discussion section below. In theory the FMF lantern should couple 6 modes for each of the 7 output legs for a total of 42 modes. Although not viable solutions for use in this scalable ground receiver concept, the specifications of SMF, graded index FMF, and 50 micron core graded index MMF are included in Table 1 for comparison.

Table 1: Photonic Lanterns and Fiber Tested

\begin{tabular}{|c|c|c|c|}
\hline Fiber & $\begin{array}{l}\text { Input/Output Core } \\
\text { Size, } \mu \mathrm{m}\end{array}$ & $\begin{array}{l}\text { InputlOutput } \\
\text { Numerical } \\
\text { Aperture }\end{array}$ & $\begin{array}{l}\# \text { of modes } \\
\text { supported }\end{array}$ \\
\hline Single-Mode Fiber & $10.4^{\mathrm{a} / 10.4^{\mathrm{a}}}$ & $0.12^{\mathrm{b}}$ & 1 \\
\hline Graded Index Few-Mode Fiber & $20 / 20$ & 0.19 & 6 \\
\hline Graded Index Multi-Mode Fiber & $50 / 50$ & 0.2 & $\sim 410$ \\
\hline 7:1 SMF lantern & $30 / 9$ & $0.08 / 0.12$ & 7 \\
\hline 7:1 FMF lantern & $40 / 20$ & $0.15 / 0.20$ & $42^{c}$ \\
\hline
\end{tabular}

\subsection{Fiber spatial mode creation with a spatial light modulator}

The spatial light modulator (SLM) was used to create the fiber spatial modes in order to test the coupling efficiency of the lanterns. The spatial modes are created by encoding phase holograms on the SLM, in which each hologram is the inverse Fourier Transform of a step index fiber spatial mode. When applied to the SLM, the phase of the Gaussian beam is modified to create the desired fiber spatial mode. The creation of the modes was verified by collecting the intensity cross section of the beam near the focus of the focusing lens with a beam profiler, as shown in Fig. 3 (b). Figure 3 shows the phase hologram for mode $\mathrm{LP}_{31}$ in (a), which was applied to achieve the intensity profile pictured in (b). This intensity profile can be compared to the theoretical intensity profile in 3(c).

Further verification on the accuracy of the mode creation was achieved by analyzing the overlap of the desired ideal mode with the created mode measured with the beam profiler. Figure 4 shows the percentage the modes created by the spatial light modulator overlap with the theoretical fiber spatial modes. In general, Fig. 4 shows that as the mode number increases, the amount of overlap decreases. This was expected due to the higher spatial frequency components in higher order modes, making holographic errors more prominent. The lowest mode overlap created was $\mathrm{LP}_{12}$ at $74.5 \%$. The highest overlap was for $\mathrm{LP}_{01}$ at $96.9 \%$.
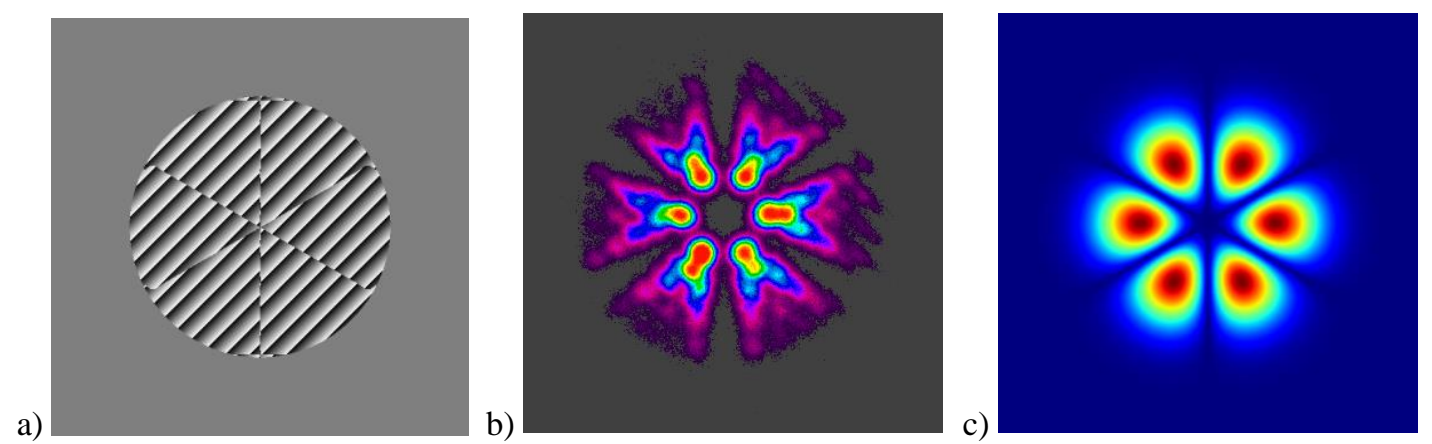

Figure 3: Fiber spatial mode $\mathrm{LP}_{31}$ creation. (a) phase hologram, (b) created intensity profile, (c) theoretical intensity profile Please note the false color scales in (b) and (c) are not the same. 


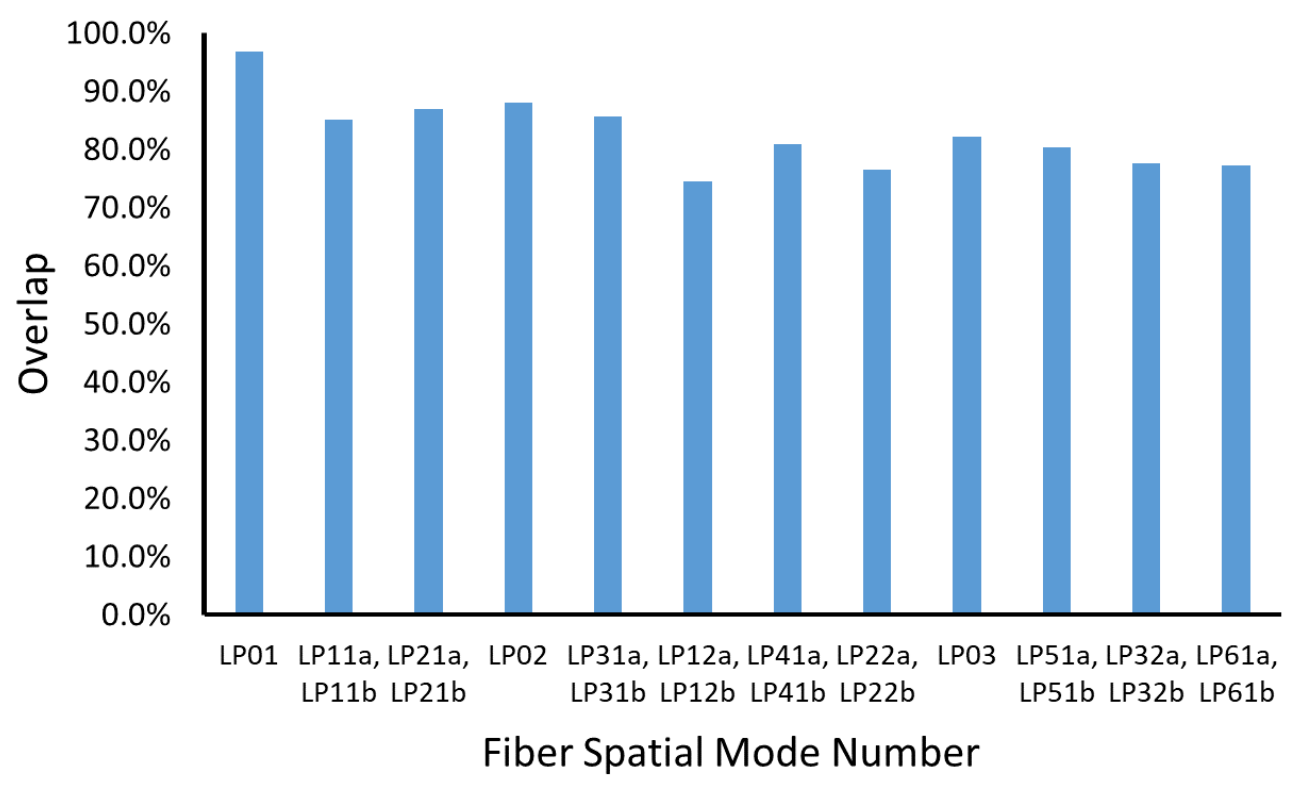

Figure 4: The percent overlap of each theoretical fiber spatial mode with the profile created by the spatial light modulator.

\section{JITTER ANAYLSIS}

In this section, the root mean square (RMS) jitter added to the system by the two different types of lanterns is analyzed and compared at $1550 \mathrm{~nm}$. For reference, the amount of RMS jitter that other fiber types would contribute are included in Table 2. The jitter is calculated using the two largest sources of pulse dispersion: differential modal delay (DMD) and chromatic dispersion. These fibers and lanterns have other smaller sources of dispersion (orders of magnitude smaller) that aren't consider here [11]. The DMD per meter reported in Table 2 are sourced from manufacture specifications and Refs. [11 and 12].

Table 2: Dispersion Effects contributed by Fibers

\begin{tabular}{lccc}
\hline \hline Fiber Device & $\begin{array}{l}\text { Differential Modal } \\
\text { Delay (ps/m) }\end{array}$ & $\begin{array}{l}\text { Chromatic } \\
\text { Dispersion } \\
(\mathbf{p s} / \mathbf{m})\end{array}$ & RMS Jitter (ps) \\
\hline Single-Mode Fiber & 0 & 0.017 & 0.145 \\
Few Mode Fiber (Graded Index) & $\pm 0.4^{\mathbf{c}}$ & 0.018 & $0.15-3.56^{\mathrm{c}}$ \\
Multimode Fiber (Graded Index) & 0.79 & 0.0245 & 6.93 \\
SMF 7:1 Lantern & Section 1:4.64, Section 2: 0 & 0.017 & 0.22 \\
FMF 7:1 Lantern & Section 1:14.00, Section 2: \pm 0.4 & 0.018 & $0.53-3.93^{\mathrm{c}}$ \\
\hline \hline
\end{tabular}

${ }^{a}$ Values reported are assumed to have a input laser linewidth of $1 \mathrm{~nm}$.

${ }^{\mathrm{b}}$ Values are calculated assuming system includes 20 meters of fiber.

${ }^{\mathrm{c}}$ The range is caused by fabrication variances from the ideal parabolic index profile.

The chromatic dispersions per meter reported in Table 2 are calculated by using:

$$
\sigma_{\text {fiber }} / \mathrm{L}=|\mathrm{D}| \sigma_{\lambda}
$$


where $\sigma \lambda$ is the laser linewidth and D is the coefficient of dispersion. For chromatic dispersion values listed in Table 2 , the laser linewidth is assumed to be $1 \mathrm{~nm}$ and the coefficients of dispersion are sourced from manufacture specifications and Ref. [11]. The last column in Table 2 reports the total added jitter to the system with the fiber length of 20 meters, which was chosen to be similar to the fiber length used in the Lunar Laser Communications Demonstration (LLCD) ground receiver [13].

The graded index FMF has DMD that varies from -0.4 ps to +0.4 ps due to fabrication variability. As explored in Ref. [14] slight variances from the ideal graded-index profile, which minimizes the DMD, results in an increased DMD. Therefore, in the worst case, a graded index FMF contributes only half as much jitter as a graded index MMF. But as shown in Ref. [14], the total DMD of the graded index fiber can be reduced by combining lengths of fiber with positive and negative DMD. Therefore, ideally, the total jitter of the graded index FMF has the potential to be reduced to chromatic dispersion only.

The photonic lanterns DMD is reported in two sections. Section 1 is the first 40 or $50 \mathrm{~mm}$ of the transition from the MMF input to the individual fibers. Section 2 is the rest of the length of the lantern made of the small core output leg fibers. For the full length of section 1 the fiber is assumed to be a MMF. The DMD per meter for section 1 is calculated using:

$$
\sigma_{\text {fiber }} / \mathrm{L}=\Delta /\left(2 \mathrm{c}_{1}\right)
$$

where $\Delta$ is $\left(\mathrm{n}_{1}-\mathrm{n}_{2}\right) / \mathrm{n}_{1}, \mathrm{c}_{1}=\mathrm{c}_{0} / \mathrm{n}_{1}, \mathrm{n}_{1}$ is the index of refraction of the core, $\mathrm{n}_{2}$ in the index of refraction of the cladding and $\mathrm{c}_{0}$ is the speed of light.

Table 2 shows that compared to MMF, a SMF lantern has a near 30 times less contribution to jitter. For the FMF lantern the worst case has 17 times more jitter than the SMF lantern. If carefully designed, with lengths of FMF with positive and negative dispersion, the FMF lantern contribution to jitter can be reduced to only $\sim 2$ times more that the SMF lantern.

\section{RESULTS AND DISCUSSION}

This section presents the coupling efficiency of lanterns for a range of NAs, mode field diameters, and fiber spatial modes for a 7:1 SMF lantern and a 7:1 FMF lantern. Also presented is how evenly the energy coupled into the lantern is split among the output fiber legs.

\subsection{Coupling Efficiency}

The coupling efficiency of the lanterns presented in this section are the ratio between the input power and the sum of the output power of all the 7 output legs. Figure 5 compares these lantern coupling efficiencies to fibers for the first 21 fiber spatial modes. The FMF couples the first 6 modes it is designed to couple at near $50 \%$ efficiency, while the remaining modes are coupled at $30 \%$ or less efficiency. Similarly, the 7:1 SMF lantern couples $\mathrm{LP}_{01}-\mathrm{LP}_{31}$ most efficiently, but then drops in efficiency by nearly 2 times for higher order modes. From this we can conjecture that any coupling efficiency in the 7:1 SMF lantern and the FMF from higher-order modes are mainly due to input crosstalk coupling due to mode field overlaps. For example, the generated $\mathrm{LP}_{03}$ mode although not guided in either the FMF or the 7:1 SMF lantern will have an strong mode field overlap (mode field size dependent) with $\mathrm{LP}_{01}$ and $\mathrm{LP}_{02}$ at the input end-face of the fibers, leading to input crosstalk coupling into these two modes.

No such dramatic change in efficiency indicating a change to crosstalk coupling is seen in the FMF lantern results. Instead, there is a gradual reduction in coupling efficiency as the spatial mode order increases. This gradual reduction in coupling efficiency can also be seen in the MMF results presented in Fig. 5 for reference only (MMF cannot efficiently deliver light to SMF or FMFs coupled detectors). This reduction can be attributed to two factors. First, as seen in Fig. 4, the accuracy of the mode creation by the SLM decreases with increasing mode order. Second, as the mode order increases, the freespace input mode field diameter increases, while the mode field diameter of the lantern (or fiber) remains the same. This decreases mode overlap at the input fiber face decreasing the energy coupled.

Despite the indistinctness of the FMF lantern spatial modes coupling capability, the coupling efficiency results are clear. The FMF lantern has similar coupling efficiency as the SMF lantern for the first 7 modes. But the FMF lantern has higher coupling efficiency at higher order modes. This indicates that at higher turbulence levels the FMF lantern will be able to couple more energy that a SMF lantern. 


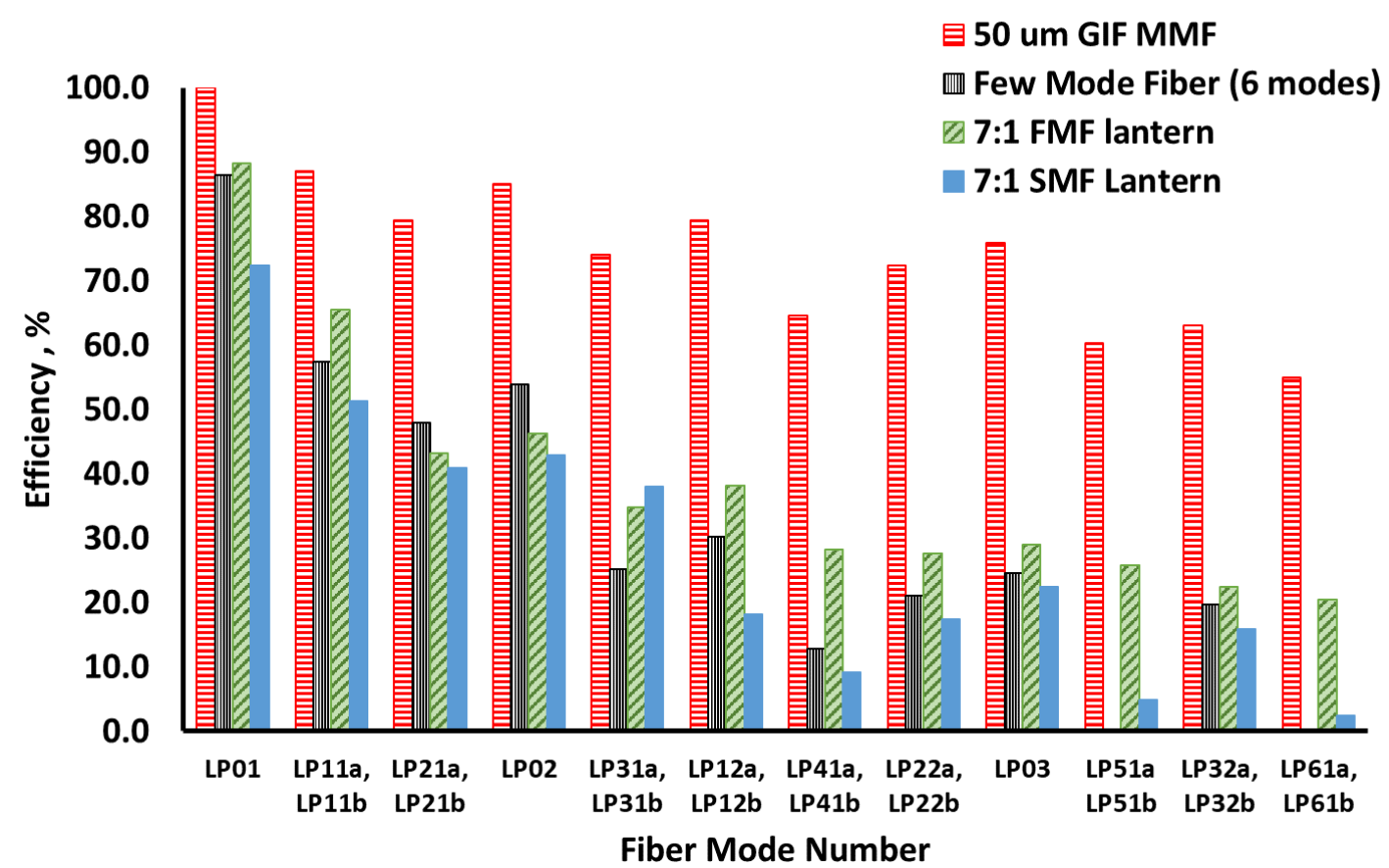

Figure 5: Coupling efficiency of the first 21 fiber spatial modes for FMF, GI-MMF, 7:1 SMF lantern, and 7:1 FMF lantern.

To assess how the lanterns couple behind a telescope, the free space coupling efficiency of the fundamental mode (LP $\left.\mathrm{P}_{01}\right)$ of the photonic lanterns were measured over a range of NAs. Figure 6 shows the dependence of the NA on the coupling efficiency of the photonic lanterns. The SMF photonic lantern (blue circles) coupling efficiency is higher than the FMF lantern (green triangles) at NA larger than 0.05. At the smallest tested NA ( 0.04), the FMF lantern is more efficient, indicating the NA is smaller for the FMF lantern than the SMF lantern.

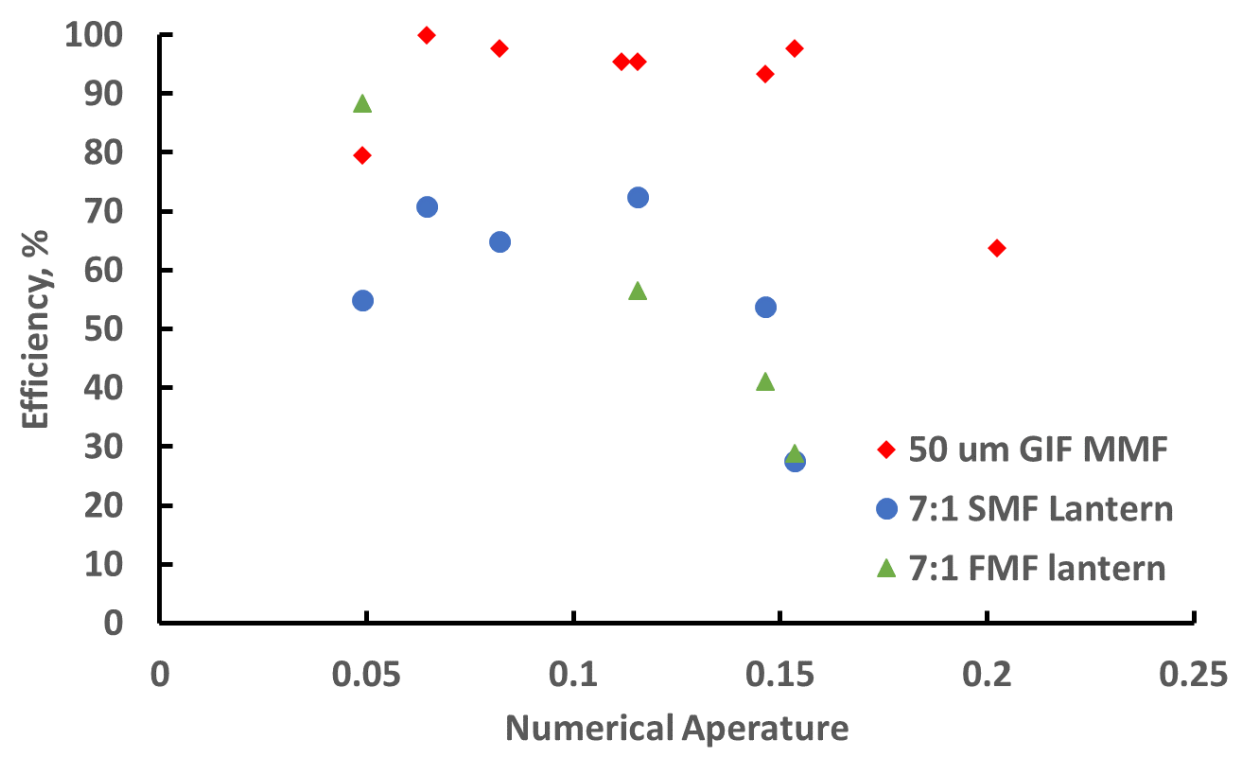

Figure 6: Plot of the free space coupling efficiency of mode $\mathrm{LP}_{01}$ (fundamental) versus numerical aperture (NA) 
Although not a viable solution for coupling to the multiple single photon 14 micron detectors, a 50 micron graded index multi-mode (GI-MMF) (red diamonds) is shown for reference. As expected, the GI-MMF has the highest efficiency for NAs greater than 0.05 . At $~ 0.04 \mathrm{NA}$, the FMF lantern outperforms as its NA is smaller than the GI-MMF. This indicates that a FMF lantern may be a more efficient solution than GI-MMF and SMF lanterns for low NA (long focal length) telescopes.

These results would have to also consider the mode field diameters of the guided $\mathrm{LP}_{01}$ modes in different fibers. In order to have an estimation, Figure 7 shows the same data but with a new perspective by converting the NA to mode field diameter (beam spot size). Again MMF and SMF lantern performs the best over the majority of the range of tested mode field diameters. The FMF lantern shows an increase of coupling efficiency at the highest mode field diameters (20 microns), indicating that for telescopes with large blur spot diameter (caused by higher turbulence conditions), FMF lantern may offer a more efficient coupling solution than SMF lantern or a MMF.

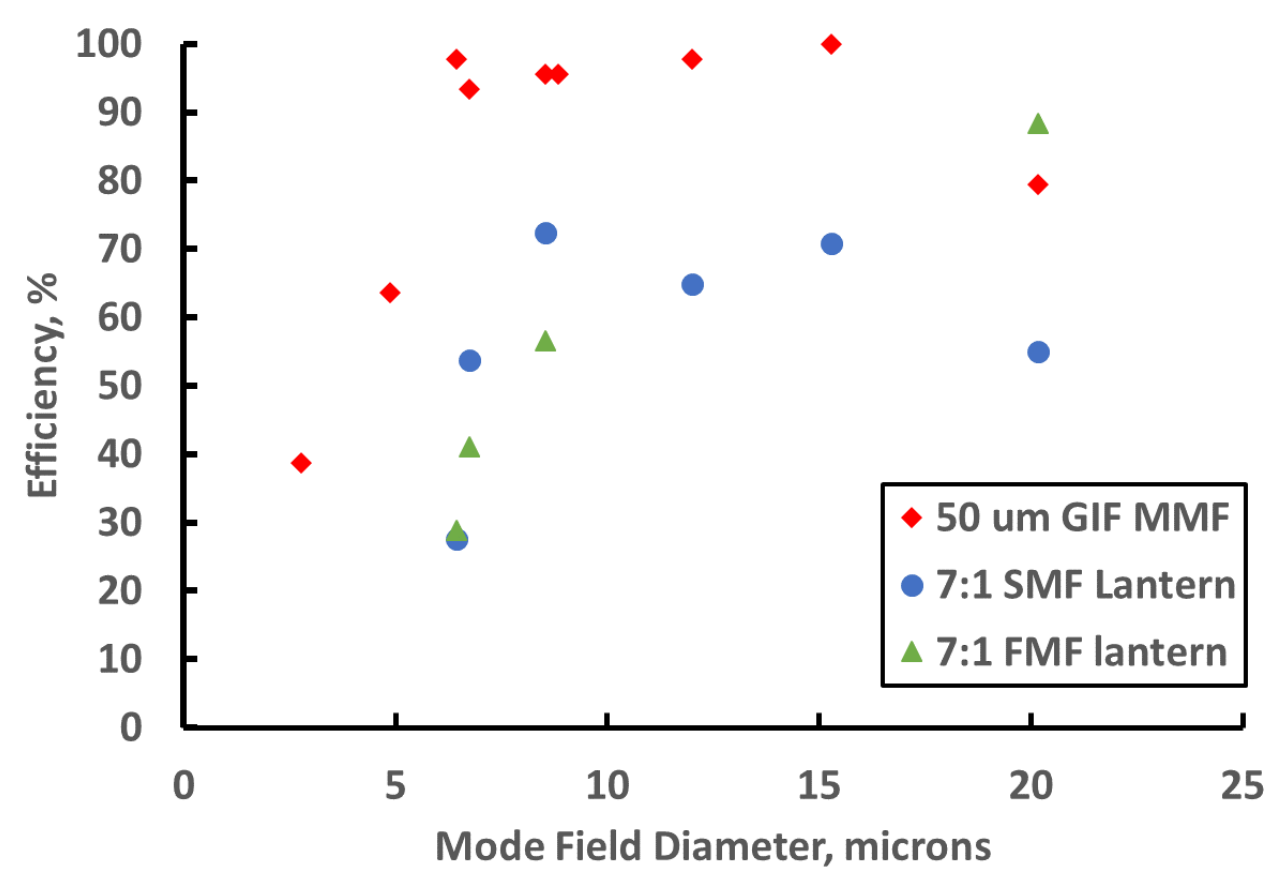

Figure 7: Plot of the free space coupling efficiency of mode $\mathrm{LP}_{01}$ (fundamental) versus mode field diameter

\subsection{Power splitting to lantern output legs}

Ideally, to achieve the least amount of detector blocking, the lanterns need to split the power evenly to each of its legs, regardless of the shape of the input beam profile. The power split of the SMF and the FMF lantern are compared in Figure 8 for 3 sample input beam profiles with the fiber mode shapes of $\mathrm{LP}_{01}, \mathrm{LP}_{11}$, and $\mathrm{LP}_{31}$. Figure 8 (a) and (b) plots the power (in terms of efficiency) for each of the seven legs for a fundamental mode input profile. The line on the plot indicates the efficiency at which each leg would be if the splitting was perfectly even. When comparing the SMF lantern and the FMF lantern, the FMF lantern shows a more even split than the SMF lantern. This is also true for the $\mathrm{LP}_{11}$ and $\mathrm{LP}_{31}$ input profiles shown in Fig 8 (c) and (d), and Fig 8 (e) and (f), accordingly. These results indicate the FMF lantern will cause less detector blocking loss than a SMF lantern. 
$\underline{\text { SMF Lantern }}$

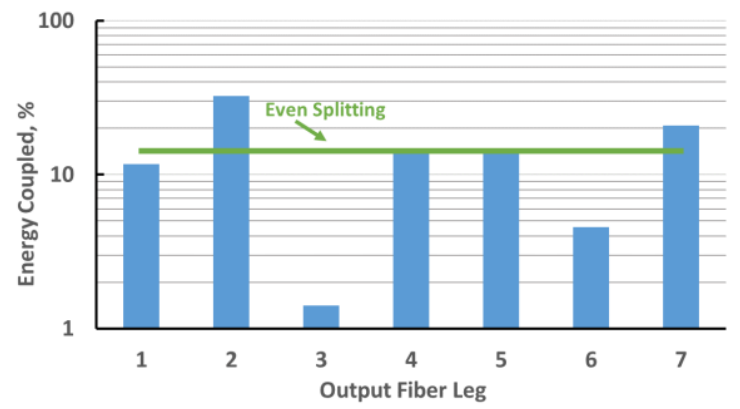

a)

c)

e)

Figure 8: Sample input beam profiles showing the power splitting (in efficiency) for the SMF lantern (a,c, and e) and FMF lantern (b,d,and f). a) and b) have an input profile of $\mathrm{LP}_{01}$ (fundamental). c) and d) have an input profile of $\mathrm{LP}_{11}$. e) and f) have an input profile of $\mathrm{LP}_{31}$

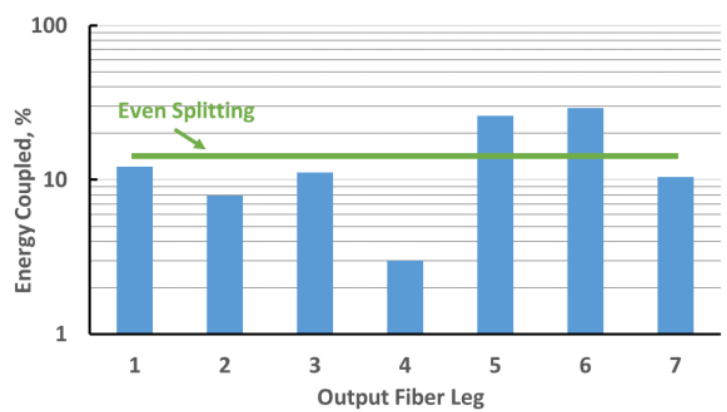

b)

d)
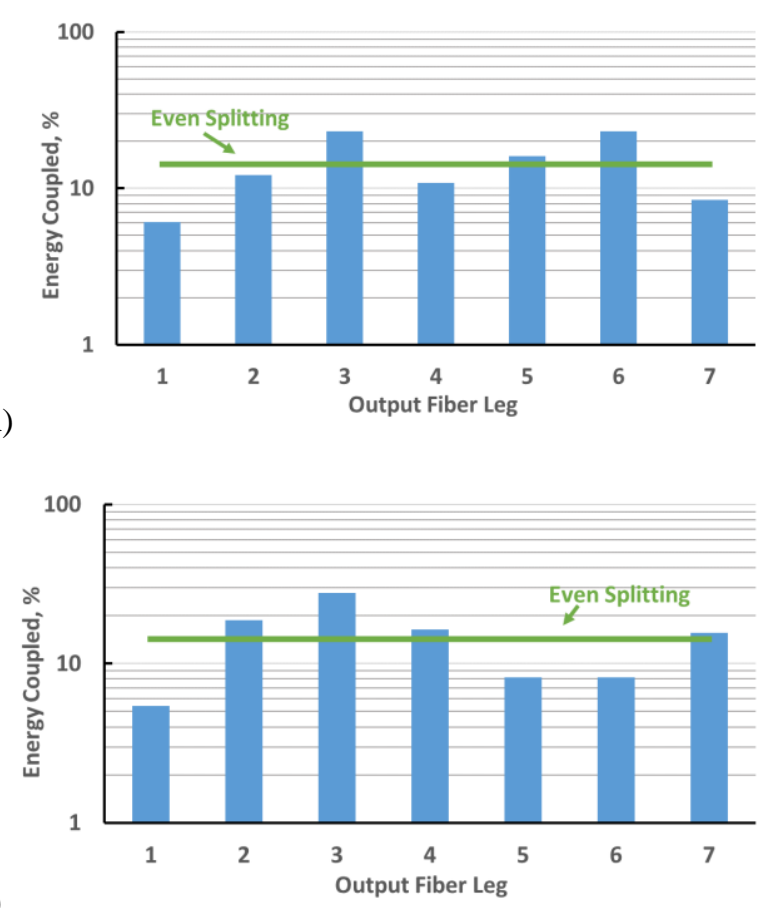

f)

\section{FMF Lantern}
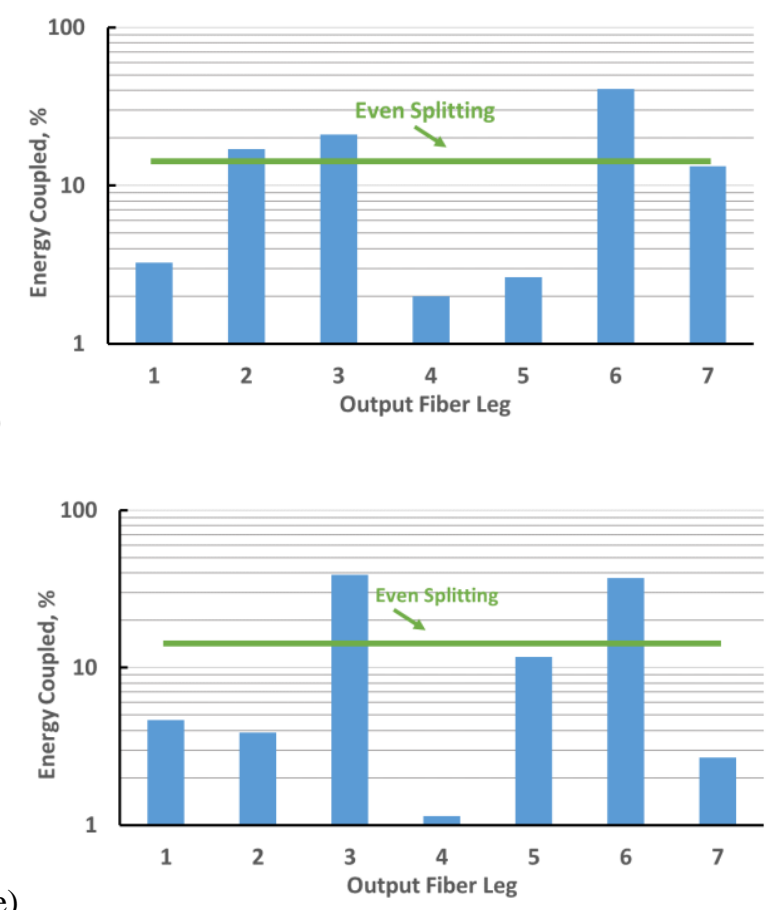

\section{CONCLUSION}

In conclusion, the preliminary results indicate that FMF lanterns are a better solution than SMF lanterns for our COTS, scalable ground receiver concept. The FMF lantern showed increased mode coupling capacity at higher-order fiber spatial modes. This indicates a capability to couple more energy at higher turbulence levels with fewer detectors than a modecontent equivalent SMF lantern system. The FMF lantern also had a higher coupling efficiency than a SMF lantern and a MMF at the smallest NA and largest mode field diameter tested. This hints that a FMF lantern may be a more efficient solution for long focal length telescopes with large blur spots. Measurements also showed that the FMF lantern power splitting is more even than the SMF lantern, thus a FMF lantern would cause less detector blocking loss in our proposed concept. The one metric in which the FMF lantern did not outperform the SMF lantern is in added jitter. The FMF lantern 
can add up to $\sim 17$ times more jitter than the SMF lantern. But this can be minimized to only $\sim 2$ times more than a SMF lantern. Further testing will have to be performed to fully characterize this behavior.

Future work will include further measurement and analysis of a FMF lantern coupled spatial modes and overall possible input crosstalk coupling between guided and unguided waveguide modes. The results presented here are measured on the first prototype of a FMF lantern. More work is needed to refine fabrication processes in hopes of improving the efficiency. Future work will also investigate other FMF lantern designs. To further understand the performance of the FMF lantern, measurements are needed in the COTS, scalable ground receiver system. Measurements will be taken of the efficiency, jitter, and detector blocking loss at a range of emulated turbulence levels. This will be executed using random phase screens on the spatial light modulator. Also needed are detailed analysis and measurement of background and polarization effects.

\section{ACKNOWLEDGEMENTS}

This work is supported by the Technology and Standards Division within NASA Space Communications and Navigation (SCaN) Program and the Communications \& Intelligent Systems Division. This work was also supported by the Sydney Astrophotonic Instrumentation Laboratory (SAIL) at the University of Sydney.

\section{REFERENCES}

[1] "Optical Communications Coding and Synchronization Draft Recommended Standard," CCSDS 142.0-R-1 Red Book (2018).

[2] Birks, T., Gris-Sanchez, I., Yerolatisitis, S., Leon-Saval, S. G., and Thompson, R. R., "The photonic lantern", Advances in Optics and Photonics, (7) (2015).

[3] Nappier, J, Vyhnalek, B., Tedder, S., and Lantz, N. "Characterization of a photon counting test bed for space to ground optical pulse position modulation communications links," Proc. SPIE Free-Space Laser Communication and Atmospheric Propagation XXXI 10910 (7) (2019).

[4] Vyhnalek, B., Tedder, S., Katz, E, and Nappier, J. "Few-mode fiber coupled superconducting nanowire single-photon detectors for photon efficient optical communications," Proc. SPIE Free-Space Laser Communication and Atmospheric Propagation XXXI 10910 (11) (2019).

[5] Ozdur, I., Toliver, P., Agarwal, A., and Woodward, T. K., "Free-space to single-mode collection efficiency enhancement using photonic lanterns", Optics Letters, Vol. 38 (18) (2013).

[6] Yarnall, T., Geisler, D., Schieler, C., and Yip, R., "Analysis of Free-Space Coupling to Photonic Lanterns in the Presence of Tilt Errors", IEEE Photonics Conference, 17393311, (2017).

[7] Geisler, D., Yarnall, T., Scheiler, C., Lund, G., Stevens, M., Robinson, B., Hamilton, S., "Ground Receiver Architectures Enabled by Digital Coherent Combing", Advanced Photonics Congress, OSA, (2018).

[8] Geisler, D., Yarnall, T., Lund, G., Schieler, C., Stevens, M., Fontaine, N., Robinson, B., and Hamilton, S., "Experimental comparison of 3-mode and single mode coupling over a 1.6-km free-space link", Proc. SPIE FreeSpace Laser Communication and Atmospheric Propagation XXX; 105240H (2018).

[9] Sergio G. Leon-Saval, Christopher H. Betters, Joel R. Salazar-Gil, Seong-Sik Min, Itandehui Gris-Sanchez, Tim A. Birks, Jon Lawrence, Roger Haynes, Dionne Haynes, Martin Roth, Sylvain Veilleux, and Joss Bland-Hawthorn, "Divide and conquer: an efficient solution to highly multimoded photonic lanterns from multicore fibres," Opt. Express 25, 17530-17540 (2017)

[10] Leon-Saval, S., Argyros, A., and Bland-Hawthorn, J., "Photonic lanterns: a study of light propagation in multimode to single-mode convertors", Optics Express, Vol. 18 (8) (2010).

[11] Saleh,B., Teich, M., [Fundamentals of Photonics], John Wiley \& Sons, Inc., Hoboken, (2007).

[12] Mori, T, Sakamoto, T., Yamamoto, T. Yamamoto, F., "Wideband WDM coherent optical MIMO transmission over 50-core GI-MMF using selective mode excitation technique", Optical Fiber Technology, 19 (2013).

[13] Grein, M. E. et al, "Design of a Ground-Based Optical Receiver for the Lunar Laser Communications Demonstration", International Conference on Space Optical Systems and Applications (2011).

[14] Gruner-Nielson, L., Sun, Y., Nicholson, J., Jakobsen, D., Jespersepn, K., Lingle, R., "Few Mode Transmission Fiber With Low DGD, Low Mode Coubling, and Low Loss", Journal of Lighwave Technology, Vol. 30. (23) (2012). 